\title{
DISTANCES IN WEIGHTED TREES AND GROUP INVERSE OF LAPLACIAN MATRICES*
}

\author{
STEPHEN J. KIRKLAND ${ }^{\dagger}$, MICHAEL NEUMANN ${ }^{\ddagger}$, AND BRYAN L. SHADER ${ }^{\S}$
}

\begin{abstract}
In this paper we find formulas for group inverses of Laplacians of weighted trees. We then develop a relationship between entries of the group inverse and various distance functions on trees. In particular, we show that the maximal and minimal entries on the diagonal of the group inverse correspond to certain pendant vertices of the tree and to a centroid of the tree, respectively. We also give a characterization for the group inverses of the Laplacian of an unweighted tree to be an $M$-matrix.
\end{abstract}

Key words. Laplacian matrix, generalized inverse, weighted tree

AMS subject classifications. Primary, 15A09; Secondary, 05C50

PII. S0895479896298713

1. Introduction. In this paper, for a weighted tree on $n$-vertices, we bring into focus the relationship between the inverse weighted generalized distance function which is defined on its vertices and the entries of the group inverse of the Laplacian matrix associated with the tree. In so doing we generalize and extend results which we have obtained in a previous paper for unweighted trees. We also shift the frame of reference for what we termed in that paper as bottleneck numbers for the tree to the inverse weighted distance function. Other results follow.

An undirected weighted graph on $n$ vertices is a graph, $\mathcal{G}$, each of whose edges $e$ has been labeled by a positive real number, $w(e)$, which is called the weight of the edge $e$. Taking the vertices of $\mathcal{G}$ to be $1,2, \ldots, n$, the Laplacian matrix of the weighted graph $\mathcal{G}$ is the $n \times n$ matrix $L=\left(\ell_{i, j}\right)$ whose $i$ th diagonal entry equals the sum of the weights of the edges incident to vertex $i$, and whose $(i, j)$ th off-diagonal entry equals 0 if there is no edge joining vertices $i$ and $j$ and equals the negative of the weight of the edge joining vertices $i$ and $j$ otherwise.

Suppose now that $\mathcal{G}$ is a weighted tree on $n$ vertices and recall that any two vertices $i$ and $j$ are joined by a unique path $\mathcal{P}_{i, j}$. We define the inverse weighted distance from vertex $i$ to vertex $j$ as the sum

$$
\tilde{d}(i, j)=\sum_{e \in \mathcal{P}_{i, j}} \frac{1}{w(e)}
$$

that is, $\tilde{d}(i, j)$ is the sum of the reciprocals of the weights of the edges on the path $\mathcal{P}_{i, j}$. We define $\tilde{d}_{i, i}=0$ for all $i=1, \ldots, n$. For any vertex $i$, we define the inverse

* Received by the editors February 12, 1996; accepted for publication (in revised form) by R. Brualdi September 13, 1996.

http://www.siam.org/journals/simax/18-4/29871.html

${ }^{\dagger}$ Department of Mathematics and Statistics, University of Regina, Regina, Saskatchewan, Canada S4S 0A2 (kirkland@max.cc.uregina.ca). This author's research was supported by NSERC grant OGP0138251.

${ }^{\ddagger}$ Department of Mathematics, University of Connecticut, Storrs, CT 06269-3009 (neumann@ math.uconn.edu). This author's research was supported by NSF grant DMS-9306357.

$\S$ Department of Mathematics, University of Wyoming, Laramie, WY 82071 (bshader@uwyo.edu). This author's research was partially supported by NSA grant MDA904-94-H-2051. 
status of vertex $i$ as the sum

$$
\tilde{d}_{i}=\sum_{u \in T} \tilde{d}(u, i)
$$

Our terminology follows that of Harary [6] for unweighted graphs.

Recall that for an $n \times n$ matrix $A$, the group inverse of $A$, when it exists, is the unique $n \times n$ matrix satisfying the matrix equations

$$
\text { (i) } A X A=A \text {, (ii) } X A X=X \text {, and (iii) } A X=X A \text {. }
$$

It is known that for real square matrix, $A$, the group inverse exists if and only if the Jordan blocks of $A$ corresponding to the eigenvalue $\lambda=0$ are all $1 \times 1$. It is customary to denote the group inverse of $A$ by $A^{\#}$.

Now let $\mathcal{G}$ be a weighted tree on $n$ vertices. It is readily seen from the definition of the Laplacian of $\mathcal{G}$ that $L$ is a symmetric matrix with nonpositive off-diagonal entries and zero row sums. It follows now from the Perron-Frobenius theory, see, for example, Berman and Plemmons [1], that $L$ is a positive semidefinite and irreducible $M$-matrix. In particular, the group inverse $L^{\#}$ of $L$ exists.

In section 3 we shall show that for some constant $c$,

$$
\left(L_{1,1}^{\#}, \ldots, L_{n, n}^{\#}\right)=\frac{1}{n}\left(\tilde{d}_{1}, \ldots, \tilde{d}_{n}\right)+c \mathbf{1},
$$

where 1 is the $n$-vector of all 1's. Thus the maximal and minimal diagonal entries in $L^{\#}$ correspond to the vertices of maximal and minimal inverse status, respectively. We shall further show that of necessity such vertices must be pendant and centroid vertices of the tree, respectively. We also find a representation in terms of the inverse status values for the off-diagonal entries of $L^{\#}$ and show that its entries corresponding to the edges along the same path emanating at any vertex decrease as we move away from the vertex. This yields a characterization for $L^{\#}$ to be an $M$-matrix itself, and we analyze the unweighted trees whose Laplacian has a group inverse which is such a matrix.

The development of distance formulas for the entries of the group inverse of the Laplacian requires the preparation of some preliminary results for weighted trees of certain parameters associated with the graph which, for unweighted trees, we called bottleneck numbers. In section 2 we extend the notion and results on bottleneck numbers in [7] to weighted graphs.

2. Formulas for $\boldsymbol{L}^{\#}$. Recall that the Laplacian matrix of a weighted connected graph is an irreducible singular $M$-matrix. In this section we establish general formulas for the group inverse of an irreducible singular $M$-matrix and then give combinatorial descriptions in the case of weighted graphs.

We begin with the following block matrix description. Let $A$ be an $n \times n$ irreducible, singular $M$-matrix. Then there exists a positive vector $x$ such that $A x=0$. The vector $x$ is called a right null vector of $A$. Similarly, a left null vector of $A$ is a positive vector $y$ such that $y^{T} A=0^{T}$. We also note, for $k=1,2, \ldots, n$, that the principle submatrix $A[\{k\},\{k\}]$ obtained from $A$ by deleting its $k$ th row and column is a nonsingular $M$-matrix. The proof of the following is similar to that of Theorem 8.5.2 in $[2]$.

Proposition 2.1. Let $A$ be an irreducible singular $M$-matrix with right null 
vector $x=\left(x_{1}, x_{2}, \ldots, x_{n}\right)^{T}$ and left null vector $y=\left(y_{1}, y_{2}, \ldots, y_{n}\right)^{T}$. Then

$$
A^{\#}=\frac{\hat{y}^{T} M \hat{x}}{\left(y^{T} x\right)^{2}} x y^{T}+\left[\begin{array}{cc}
M-\frac{1}{y^{T} x} M \hat{x} \hat{y}^{T}-\frac{1}{y^{T} x} \hat{x} \hat{y}^{T} M & \frac{-y_{n}}{y^{T} x} M \hat{x} \\
-x_{n} \hat{y}^{T} M & 0
\end{array}\right],
$$

where $\hat{x}=\left(x_{1}, x_{2}, \ldots, x_{n-1}\right)^{T}, \hat{y}=\left(y_{1}, y_{2}, \ldots, y_{n-1}\right)^{T}$, and $M=A[\{n\},\{n\}]^{-1}$.

If $A$ is an $n \times n$ irreducible singular $M$-matrix, we call the nonnegative matrix $A[\{i\},\{i\}]^{-1}$ the bottleneck matrix of $A$ based at $i$. Proposition 2.1 describes $A^{\#}$ in terms of the bottleneck matrix of $A$ based at $n$. If $A$ is the Laplacian matrix of a weighted graph $\mathcal{G}$, then we also refer to $A[\{i\},\{i\}]^{-1}$ as the bottleneck matrix of $\mathcal{G}$ based at vertex $i$. The following is an immediate consequence of Proposition 2.1 and the fact that $\mathbf{1}$ is a left and right null vector of the Laplacian matrix of a weighted graph.

Proposition 2.2. Let $L$ be the Laplacian matrix of a connected weighted graph with $n$ vertices. Then

$$
L^{\#}=\frac{\mathbf{1}^{T} M \mathbf{1}}{n^{2}} J+\left[\begin{array}{cc}
M-{ }_{n}^{1} M J-{ }_{n}^{1} J M & -{ }_{n}^{1} M \mathbf{1} \\
-{ }_{n}^{1} \mathbf{1}^{T} M & 0
\end{array}\right],
$$

where $M=L[\{n\},\{n\}]^{-1}$ is the bottleneck matrix based at vertex $n$.

If $A$ is an $m \times n$ matrix, the submatrix of $A$ whose rows have index in $\alpha$ and whose columns have index in $\beta$ is denoted by $A[\alpha, \beta]$. We use $\alpha$ and $\beta$ to denote the complement of $\alpha$ in $\{1,2, \ldots, m\}$ and of $\beta$ in $\{1,2, \ldots, n\}$, respectively.

We now develop a combinatorial description of the entries of the bottleneck matrix of a weighted graph. Let $L$ be the Laplacian matrix of a connected weighted graph $\mathcal{G}$ with vertices $1,2, \ldots, n$. By the cofactor formula for the inverse, the $(i, j)$ th entry of the bottleneck matrix of $L$ based at $n$ equals

$$
\begin{gathered}
(-1)^{i+j} \operatorname{det} L[\{j, n\},\{i, n\}] . \\
\operatorname{det} L[\{n\},\{n\}]
\end{gathered}
$$

Our description of the bottleneck matrix follows from (2.1) and a generalization of the matrix-tree theorem obtained by Chaiken in [3]. The generalization is proven using the Cauchy-Binet formulas and gives a combinatorial description of the determinants of the square submatrices of a Laplacian matrix.

For the purpose of achieving the above, we need a few further graph theoretical notions. A subgraph of $\mathcal{G}$ is a graph $\mathcal{H}$ whose vertices are a subset of $1,2, \ldots, n$, and whose edges are a subset of those of $\mathcal{G}$. If $\mathcal{G}$ is a weighted graph and $E$ is a subset of edges of $\mathcal{G}$, then the weight of $E$ is denoted by $w(e)$ and is the product of the weights of the edges in $E$. The weight of a subgraph $\mathcal{H}$ is the weight of its set of edges and the weight of a graph with no edges is defined to be 1 . The set of all spanning trees of $\mathcal{G}$ is denoted by $\mathcal{S}$. We now define a special type of spanning forest. Let $i, j$, and $k$ be (not necessarily distinct) vertices of $\mathcal{G}$. An $(\{i, j\}, k)$-spanning forest of $\mathcal{G}$ is a spanning forest of $\mathcal{G}$ which has exactly two connected components, one of which contains vertex $k$ and the other of which contains the vertices $i$ and $j$. The set of all $(\{i, j\}, k)$-spanning forests of $\mathcal{G}$ is denoted by $\mathcal{S}_{k}^{\{i, j\}}$. 
Chaiken's all minors matrix tree theorem in [3] implies that

$$
\operatorname{det} L[\{n\},\{n\}]=\sum_{T \in \mathcal{S}} w(T)
$$

and

$$
\operatorname{det} L[\{j, n\},\{i, n\}]=\sum_{F \in \mathcal{S}_{v}^{\{i, j\}}} w(F) \quad \text { for } 1 \leq i, j \leq n-1 .
$$

Therefore, (2.1) implies the following theorem.

TheOREM 2.3. Let $\mathcal{G}$ be a connected weighted graph on vertices $1,2, \ldots, n$. Let $v$ be a vertex of $\mathcal{G}$. Then the bottleneck matrix $M=\left[m_{i, j}\right]$ of $\mathcal{G}$ based at vertex $v$ satisfies

$$
m_{i, j}=\frac{\sum_{F \in \mathcal{S}_{v}^{\{i, j\}}} w(F)}{\sum_{T \in \mathcal{S}} w(T)} .
$$

Theorem 2.3 has numerous consequences. The first, which is immediate, is a combinatorial formula for the bottleneck matrix of an unweighted graph.

COROLLARY 2.4. Let $\mathcal{G}$ be an unweighted connected graph with $n$ vertices. Then the bottleneck matrix, $M=\left[m_{i, j}\right]$, based at vertex $v$ satisfies

$$
m_{i, j}=\frac{\left|\mathcal{S}_{v}^{\{i, j\}}\right|}{|\mathcal{S}|} .
$$

Let $\mathcal{G}$ be an unweighted connected graph with vertices $1,2, \ldots, n$. Let $i$ and $j$ be adjacent vertices. Adding the edge joining $i$ and $j$ to each forest in $\mathcal{S}_{j}^{\{i, i\}}$ establishes a correspondence between $\mathcal{S}_{n}^{\{i, i\}}$ and the spanning trees of $\mathcal{G}$ which contain the edge joining $i$ and $j$. Hence, by Corollary 2.4, the $(i, i)$-entry of the bottleneck matrix of $\mathcal{G}$ based at vertex $j$ is equal to the fraction of spanning trees of $\mathcal{G}$ which contain the edge joining $i$ and $j$. An analogous result holds for weighted graphs and follows from Theorem 2.3 .

Let $T$ be a weighted tree with vertices $1,2, \ldots, n$. Let $i$ and $j$ be (not necessarily distinct) vertices other than $n$ of $T$. Each spanning forest $F$ of $T$ with exactly two components can be obtained from $T$ by removing exactly one edge $e$. Thus, a spanning forest $F$ of $T$ is an $(\{i, j\}, n)$-spanning forest if and only if $F$ is a spanning forest obtained from $T$ by removing one of the edges $e$ which lies on the path from $i$ to $n$ and on the path from $j$ to $n$. Since $\frac{w(F)}{w(T)}=\underset{w(e)}{1}$, Theorem 2.3 implies the following.

Corollary 2.5. Let $T$ be a weighted tree with vertices $1,2, \ldots, n$. The bottleneck matrix, $M=\left[m_{i, j}\right]$, of $\mathcal{G}$ based at vertex $v$ satisfies

$$
m_{i, j}=\sum_{e \in \mathcal{P}_{i, j}^{v}} \frac{1}{w(e)}
$$

where $\mathcal{P}_{i, j}^{v}$ is the set of all edges e which lie on the path from $i$ to $v$ and on the path from $j$ to $v$.

A direct proof of Corollary 2.5 is given in [8]. Note that if $T$ is an unweighted tree, then by Corollary 2.5, $m_{i, j}=\left|\mathcal{P}_{i, j}^{v}\right|$. This was shown in [7]. 
Let $A=\left[a_{i, j}\right]$ be an $n \times n$ matrix. The matrix $A$ is combinatorially symmetric provided $a_{i, j}=0$ whenever $a_{j, i}=0$. If $A$ is combinatorially symmetric, then the graph of $A$ is the graph $\mathcal{G}$ with vertices $1,2, \ldots, n$ with an edge joining vertex $i$ and vertex $j$ if and only if $i \neq j$ and $a_{i, j} \neq 0$. If $A$ is symmetric, then the weighted graph of $A$ is the graph obtained from $\mathcal{G}$ by weighting the edge joining vertex $i$ and $j$ by $a_{i, j}$. The next result extends Corollary 2.5 to symmetric $M$-matrices whose graph is a tree.

COROLlary 2.6. Let $A$ be a symmetric, singular $M$-matrix whose graph is a tree $T$ with $n$ vertices. Let $x$ be a right null vector of $A$. Then the bottleneck matrix, $M=\left[m_{i, j}\right]$, of $A$ based at $v$ satisfies

$$
m_{i, j}=x_{i} x_{j} \sum_{e=\{k, \ell\} \in \mathcal{P}_{i, j}^{v}} \frac{1}{w(e) x_{k} x_{\ell}} .
$$

Proof. Without loss of generality we take $v=n$. Let $D$ be the $n \times n$ diagonal matrix whose diagonal entries are the entries of $x$. Consider the matrix $L=D A D$. It is easy to verify that $L$ is the Laplacian matrix of the weighted tree obtained from $T$ by weighting the edge $e$ by $w(e)=w(e) x_{k} x_{\ell}$, where $k$ and $\ell$ are the vertices incident to $e$. Hence, by Corollary 2.5,

$$
(L[\{n\},\{n\}])^{-1}=\sum_{e \in \mathcal{P}_{i, j}^{n}} \frac{1}{w(e)}=\sum_{e=\{k, \ell\} \in \mathcal{P}_{i, j}^{n}} \frac{1}{w(e) x_{k} x_{\ell}} .
$$

The corollary now follows from the observation that

$$
A[\{n\},\{n\}]^{-1}=\hat{D}(L[\{n\},\{n\}])^{-1} \hat{D}
$$

where $\hat{D}$ is the diagonal matrix obtained from $D$ by deleting row and column $n$.

Corollary 2.6 can be extended to combinatorially symmetric singular $M$-matrices whose graph is a tree. First, it is shown that every such matrix is diagonally similar to a symmetric matrix. This implies that in studying the diagonal entries of the group inverse of a combinatorially symmetric singular $M$-matrix, one may assume that the matrix is in fact symmetric. The following lemma is, essentially, due to Parter and Youngs [10], though the proof below is different.

Lemma 2.7 (see Parter and Youngs [10, Lemma 3]). Let $A$ be a combinatorially symmetric, singular $M$-matrix of order $n$ whose graph is a tree $T$. Let $x=\left(x_{1}, x_{2}, \ldots, x_{n}\right)^{T}$ and $y=\left(y_{1}, y_{2}, \ldots, y_{n}\right)^{T}$ be right and left null vectors of $A$, respectively. Let $D=\operatorname{diag}\left(\frac{\sqrt{x_{1}}}{\sqrt{ } y_{1}}, \sqrt{x_{2}}, \ldots, \frac{\sqrt{x_{n}}}{\sqrt{ } y_{n}}\right)$. Then $D^{-1} A D$ is a symmetric, singular $M$-matrix whose graph is T.

Proof. The proof is by induction on $n$. The result is clearly true if $n=1$ or $n=2$. Now assume that $n \geq 3$, and the result is true for any combinatorially symmetric, singular $M$-matrix of order $n-1$ whose graph is a tree. Consider the $n \times n$ matrix $A=\left[a_{i, j}\right]$ and its graph $T$. Without loss of generality we may assume that vertex $n$ is a pendant vertex in $T$ and is adjacent to vertex $n-1$. Since $A x=0$ and $y^{T} A=0$,

$$
\frac{y_{n-1}}{y_{n}} a_{n-1, n}=-a_{n, n}=\frac{x_{n-1}}{x_{n}} a_{n, n-1} .
$$

Thus, it follows that the last row and column of $D^{-1} A D$ are transposes of each other. The matrix

$$
\hat{A}=A[\{n\},\{n\}]-\frac{x_{n}}{x_{n-1}} a_{n-1, n} E_{n-1, n-1},
$$


where $E_{n-1, n-1}$ is the $n-1 \times n-1$ matrix with a 1 in position $(n-1, n-1)$ and 0 elsewhere, is a singular $M$-matrix whose graph is the tree obtained from $T$ by deleting vertex $n$. In addition,

$$
\hat{A}\left[\begin{array}{c}
x_{1} \\
x_{2} \\
\vdots \\
x_{n-1}
\end{array}\right]=0 \text { and }\left[\begin{array}{llll}
y_{1} & y_{2} & \cdots & y_{n-1}
\end{array}\right] \hat{A}=0 .
$$

Thus, it follows by induction that $\hat{D}^{-1} \hat{A} \hat{D}$ is symmetric where

$$
\hat{D}=\operatorname{diag}\left(\begin{array}{l}
\sqrt{ } x_{1} \\
\sqrt{ } y_{1}
\end{array}, \sqrt{x_{2}}, \ldots, \frac{\sqrt{ } x_{n-1}}{\sqrt{ } y_{n-1}}\right) .
$$

Since $\hat{D}^{-1} A[\{n\},\{n\}] \hat{D}=\left(D^{-1} A D\right)[\{n\},\{n\}]$, we conclude that $D^{-1} A D$ is symmetric.

COROllary 2.8. Let $A$ be an $n \times n$ combinatorially symmetric, singular $M$ matrix whose graph is a tree $T$ and whose right and left null vectors are $x=\left(x_{1}, x_{2}, \ldots, x_{n}\right)^{T}$ and $y=\left(y_{1}, y_{2}, \ldots, y_{n}\right)^{T}$, respectively. Then the bottleneck matrix $M=\left[m_{i, j}\right]$ of $A$ based at $v$ satisfies

$$
m_{i, j}=x_{j} y_{i} \sum_{e=\{k, \ell\} \in \mathcal{P}_{i, j}^{v}} \frac{1}{a_{k \ell} x_{\ell} y_{k}} .
$$

Proof. Without loss of generality we assume that $v=n$. Let $B=D^{-1} A D$, where $D=\operatorname{diag}\left({ }_{\sqrt{ } y_{1}}, \sqrt{x_{2}}, \ldots, \frac{\sqrt{ } x_{n}}{\sqrt{ } y_{2}}\right)$. By Lemma $2.7, B=\left[b_{i, j}\right]$ is a symmetric, singular $M$-matrix whose graph is $T$. It is easy to verify that

$$
z=\left(\sqrt{ } x_{1} y_{1}, \sqrt{ } x_{2} y_{2}, \ldots, \sqrt{ } x_{n} y_{n}\right)^{T}
$$

is a right null vector of $B$, and that the weight of an edge joining vertices $k$ and $\ell$ equals $a_{k \ell} \underset{\sqrt{ } x_{k} y_{\ell} y_{\ell}}{\sqrt{x_{1}}}$. Hence by Corollary 2.6, the $(i, j)$-entry of $(B[\{n\},\{n\}])^{-1}$ equals

$$
\sqrt{ } x_{i} y_{i} \sqrt{ } x_{j} y_{j} \sum_{e=\{k, \ell\} \in \mathcal{P}_{i, j}} \frac{1}{\left(a_{k \ell} \underset{\sqrt{ } x_{k} y_{\ell}}{\sqrt{ } y_{\ell} y_{k}} \sqrt{x_{k} y_{k}} \sqrt{ } x_{\ell} y_{\ell}\right)},
$$

which simplifies to

$$
\sqrt{ } x_{i} x_{j} y_{i} y_{j} \sum_{e=\{k, \ell\} \in \mathcal{P}_{i, j}} \frac{1}{a_{k, \ell} x_{\ell} y_{k}} .
$$

Since $B[\{n\},\{n\}]=\hat{D}^{-1} A[\{n\},\{n\}] \hat{D}$, where $\hat{D}=D[\{n\},\{n\}]$,

$$
m_{i, j}=x_{j} y_{i} \sum_{e=\{k, \ell\} \in \mathcal{P}_{i, j}} \frac{1}{a_{k, \ell} x_{\ell} y_{k}} .
$$

Next we derive a formula for the diagonal entries of Laplacians of trees. Let $T$ be a tree. If $e$ is an edge, then $T \backslash e$ denotes the graph obtained from $T$ by removing 
$e$. If $i$ is a vertex of $T$, then we define $\beta_{i}(e)$ to be the set of vertices in the connected component of $T \backslash e$ which does not contain vertex $i$.

Lemma 2.9. Let $A=\left[a_{i, j}\right]$ be an $n \times n$ symmetric, singular $M$-matrix whose graph is a tree $T$ and let $x=\left(x_{1}, x_{2}, \ldots, x_{n}\right)^{T}$ be a null vector of $A$. Then, for $v=1,2, \ldots, n$,

$$
A_{v, v}^{\#}=\frac{\left(x_{v}\right)^{2}}{\left(x^{T} x\right)^{2}} \sum_{e=\{k, \ell\} \in T} \frac{1}{w(e) x_{k} x_{\ell}}\left(\sum_{i \in \beta_{v}(e)} x_{i}^{2}\right)^{2} .
$$

Proof. Without loss of generality we may assume that $v=n$. By Proposition 2.1,

$$
A_{n, n}^{\#}=\frac{\left(x_{n}\right)^{2}}{\left(x^{T} x\right)^{2}} \hat{x}^{T} M \hat{x}
$$

where $\hat{x}$ is the vector obtained from $x$ by deleting its last row. Therefore, by Corollary 2.6 ,

$$
\begin{aligned}
A_{n, n}^{\#} & =\frac{\left(x_{n}\right)^{2}}{\left(x^{T} x\right)^{2}}\left(\sum_{1 \leq i, j \leq n-1} x_{i}^{2} x_{j}^{2} \sum_{e=\{k, \ell\} \in \mathcal{P}_{i, j}^{n}} \frac{1}{} w(e) x_{k} x_{\ell}\right) \\
& =\frac{\left(x_{n}\right)^{2}}{\left(x^{T} x\right)^{2}} \sum_{e \in T} \frac{1}{w(e) x_{k} x_{\ell}}\left(\sum_{\substack{1 \leq i, j \leq n-1 \\
e \in \mathcal{P}_{i, j}^{n}}} x_{i}^{2} x_{j}^{2}\right) \\
& =\frac{\left(x_{n}\right)^{2}}{\left(x^{T} x\right)^{2}} \sum_{e \in T} \frac{1}{w(e) x_{k} x_{\ell}}\left(\sum_{i \in \beta_{n}(e)} x_{i}^{2}\right)^{2} .
\end{aligned}
$$

The last equality follows from the fact that $i$ and $j$ are vertices such that $e \in \mathcal{P}_{i, j}^{n}$ if and only if both $i$ and $j$ belong to $\beta_{n}(e)$.

We have the following immediate consequence for the Laplacian matrix of a weighted tree. The analogous result for the Laplacian matrix of an unweighted tree is contained in Theorem 3.3 of [7].

COROLlary 2.10. If $L$ is the Laplacian matrix of a weighted tree $T$ with $n$ vertices, then

$$
L_{v, v}^{\#}=\frac{1}{n^{2}} \sum_{e \in T} \frac{\left|\beta_{v}(e)\right|^{2}}{w(e)}
$$

for $v=1,2, \ldots, n$.

The analogous result for nonsymmetric matrices follows by a similar argument.

COROLlaRY 2.11. If $A$ is an $n \times n$ combinatorially symmetric, singular $M$-matrix whose graph is a tree and $x$ and $y$ are right and left null vectors, respectively, of $A$, then

$$
A_{v, v}^{\#}=\frac{x_{v} y_{v}}{\left(y^{T} x\right)^{2}} \sum_{e=\{k, \ell\} \in T} \frac{1}{a_{k, \ell} x_{\ell} y_{k}}\left(\sum_{i \in \beta_{v}(e)} x_{i} y_{i}\right)^{2}
$$


for $v=1,2, \ldots, n$.

We now give a formula for the difference between certain diagonal entries.

Lemma 2.12. Let $A=\left[a_{i j}\right]$ be an $n \times n$ symmetric, singular $M$-matrix whose graph is a tree $T$ and let $x=\left(x_{1}, x_{2}, \ldots, x_{n}\right)^{T}$ be the null vector of $A$. Assume that vertices $i$ and $j$ are joined by an edge $e$. Then

$$
\frac{1}{x_{i}^{2}} A_{i, i}^{\#}-\frac{1}{x_{j}^{2}} A_{j, j}^{\#}=\frac{1}{\left(x^{T} x\right)^{2}} \frac{1}{w(e) x_{i} x_{j}}\left[\left(\sum_{k \in \beta_{i}(e)} x_{k}^{2}\right)^{2}-\left(\sum_{k \in \beta_{j}(e)} x_{k}^{2}\right)^{2}\right] .
$$

Proof. Let $f$ be an edge of $T$ with $f \neq e$. Then $\beta_{j}(f)=\beta_{i}(f)$. The result now follows from Lemma 2.9.

For weighted trees we have the following lemma.

Lemma 2.13. Let $T$ be a weighted tree on $n$ vertices with Laplacian matrix $L$. Suppose that $i$ and $j$ are vertices of $T$ joined by the edge $e$. Then

$$
L_{i, i}^{\#}-L_{j, j}^{\#}=\frac{1}{n w(e)}\left(\left|\beta_{i}(e)\right|-\left|\beta_{j}(e)\right|\right) .
$$

In particular, $L_{i, i}^{\#}>L_{j, j}^{\#}$ if and only if $\left|\beta_{i}(e)\right|>\left|\beta_{j}(e)\right|$.

Proof. Since 1 is a null vector of $L$, and $\left|\beta_{i}(e)\right|+\left|\beta_{j}(e)\right|=n$, the result follows from Lemma 2.12.

Finally, in the next section we shall also require a formula for the off-diagonal entries of the Laplacian of a weighted tree.

Lemma 2.14. Let $T$ be a weighted tree on $n$ vertices with Laplacian matrix $L$. Then, for $i, j=1, \ldots, n$ with $i \neq j$,

$$
L_{i, j}^{\#}=\frac{1}{n^{2}} \sum_{e \in T} \frac{\left|\beta_{j}(e)\right|^{2}}{w(e)}-\frac{1}{n} \sum_{e \in \mathcal{P}_{i, j}} \frac{\left|\beta_{j}(e)\right|}{w(e)} .
$$

Proof. Without loss of generality we can assume that $j=n$ and $i=1, \ldots, n-1$. Then using the symmetry of $L$, it follows from Proposition 2.2 and Corollary 2.6 that

$$
\begin{aligned}
L_{n, i}^{\#} & =\frac{1}{n^{2}} \mathbf{1}^{T} M \mathbf{1}-\frac{1}{n} \sum_{k=1}^{n-1} \sum_{e \in \mathcal{P}_{i, k}^{n}} \frac{1}{w(e)} \\
& =\frac{1}{n^{2}} \sum_{e \in T} \frac{\left|\beta_{j}(e)\right|^{2}}{w(e)}-\frac{1}{n} \sum_{e \in \mathcal{P}_{i, n}} \frac{\left|\beta_{i}(e)\right|}{w(e)} .
\end{aligned}
$$

3. Inverse weighted distances. We begin with the following auxilliary lemma. Lemma 3.1. Let $T$ be a weighted tree on $n$ vertices. Let $v_{0}$ and $v_{l}$ be vertices in $T$ with $v_{1}, v_{2}, \ldots, v_{l-1}$ as intermediate vertices on the path $\alpha$ which joins $v_{0}$ to $v_{l}$. For $1 \leq i \leq l$, let $e_{i}$ be the edge between $v_{i-1}$ and $v_{i}$ having weight $\theta_{i}$. For $0 \leq i \leq l$, let $t_{i}$ be the number of vertices, including $v_{i}$ whose shortest path to $\alpha$ has terminal vertex $v_{i}$. Then

$$
\tilde{d}_{v_{0}}-\tilde{d}_{v_{l}}=\sum_{i=0}^{l} t_{i}\left(\sum_{m=1}^{i} \frac{1}{\theta_{m}}-\sum_{m=i+1}^{l} \frac{1}{\theta_{m}}\right) .
$$


Proof. For any one of the $t_{i}$ vertices $u$ of $T$ whose shortest path to $\alpha$ ends at $v_{i}$, we have that

$$
\tilde{d}\left(u, v_{0}\right)-\tilde{d}\left(u, v_{l}\right)=\tilde{d}\left(v_{i}, v_{0}\right)-\tilde{d}\left(v_{i}, v_{l}\right) .
$$

Hence we find that

$$
\tilde{d}_{v_{0}}-\tilde{d}_{v_{l}}=\sum_{i=1}^{l} t_{i}\left[\tilde{d}\left(v_{i}, v_{0}\right)-\tilde{d}\left(v_{i}, v_{l}\right)\right] .
$$

The result now follows on observing that

$$
\tilde{d}\left(v_{i}, v_{0}\right)-\tilde{d}\left(v_{i}, v_{l}\right)=\left(\sum_{m=1}^{i} \frac{1}{\theta_{m}}-\sum_{m=i+1}^{l} \frac{1}{\theta_{m}}\right) .
$$

Lemma 3.1 leads us to the following theorem.

THEOREM 3.2. Under the assumptions and notations of Lemma 3.1 we have that

$$
n\left(L_{v_{0}, v_{0}}^{\#}-L_{v_{l}, v_{l}}^{\#}\right)=\sum_{i=0}^{l} t_{i}\left(\sum_{m=1}^{i} \frac{1}{\theta_{m}}-\sum_{m=i+1}^{l} \frac{1}{\theta_{m}}\right) .
$$

Proof. From (2.2) we have that

$$
n^{2} L_{v_{0}, v_{0}}^{\#}=\sum_{e \in T} \frac{\beta_{v_{0}}^{2}(e)}{w(e)}
$$

and that

$$
n^{2} L_{v_{l}, v_{l}}^{\#}=\sum_{e \in T} \frac{\beta_{v_{l}}^{2}(e) \mid}{w(e)}
$$

Now if $e \notin \alpha$, then $\left|\beta_{v_{0}}(e)\right|=\left|\beta_{v_{l}}(e)\right|$, while if $e=e_{m}$ for some $m=1,2, \ldots, \ell$, then

$$
\left|\beta_{v_{0}}\left(e_{m}\right)\right|=t_{m}+\cdots+t_{l}
$$

and

$$
\left|\beta_{v_{l}}\left(e_{m}\right)\right|=t_{0}+\cdots+t_{m-1} .
$$

Hence,

$$
\begin{aligned}
n^{2}\left(L_{v_{0}, v_{0}}^{\#}-L_{v_{l}, v_{l}}^{\#}\right) & =\sum_{m=1}^{l}\left(\left|\beta_{v_{0}}\left(e_{m}\right)\right|+\left|\beta_{v_{l}}\left(e_{m}\right)\right|\right) \frac{\left|\beta_{v_{0}}\left(e_{m}\right)\right|-\left|\beta_{v_{l}}\left(e_{m}\right)\right|}{w\left(e_{m}\right)} \\
& =n \sum_{m=1}^{l} \frac{-t_{0}-\cdots-t_{m-1}+t_{m}+\cdots+t_{l}}{\theta_{m}} .
\end{aligned}
$$

In the above sum, we collect terms in each $t_{i}$ to find that

$$
n^{2}\left(L_{v_{0}, v_{0}}^{\#}-L_{v_{l}, v_{l}}^{\#}\right)=n \sum_{i=0}^{l} t_{i}\left(\sum_{m=1}^{i} \frac{1}{\theta_{m}}-\sum_{m=i+1}^{l} \frac{1}{\theta_{m}}\right) .
$$


Corollary 3.3. Let $T$ be a weighted tree on $n$ vertices and $L$ be its Laplacian. Then for some constant $c$,

$$
\left(L_{1,1}^{\#}, \ldots, L_{n, n}^{\#}\right)=\frac{1}{n}\left(\tilde{d}_{1}, \ldots, \tilde{d}_{n}\right)+c \mathbf{1} .
$$

Proof. Let $Z$ be the simple cycle permutation matrix sending $1 \rightarrow n$ and $i \rightarrow$ $i-1, i=2, \ldots, n$. It follows from Lemma 3.1 and Theorem 3.2 that

$$
(I-Z)\left[\left(L_{1,1}^{\#}, \ldots, L_{n, n}^{\#}\right)^{T}-\frac{1}{n}\left(\tilde{d}_{1}, \ldots, \tilde{d}_{n}\right)^{T}\right]=0
$$

from which the conclusion easily follows.

This corollary shows that if $i_{1}, \ldots, i_{n}$ are indices of entries in $\left(L_{1,1}^{\#}, \ldots, L_{n, n}^{\#}\right)^{T}$ such that

$$
L_{i_{1}, i_{1}}^{\#} \geq L_{i_{2}, i_{2}}^{\#} \geq \cdots \geq L_{i_{n}, i_{n}}^{\#}
$$

then

$$
\tilde{d}_{i_{1}, i_{1}} \geq \tilde{d}_{i_{2}, i_{2}} \geq \cdots \geq \tilde{d}_{i_{n}, i_{n}} .
$$

In particular we have the following conclusion.

Corollary 3.4. Let $T$ be a weighted tree on $n$ vertices and $L$ its Laplacian. Then a diagonal entry in $L^{\#}$ occurs at an index which corresponds to an index of a pendant vertex of $T$ whose inverse status is maximal and a minimal diagonal entry in $L^{\#}$ occurs at an index which corresponds to vertex of $T$ which is a centroid.

Proof. To prove the first part of the corollary, suppose that $k$ is not a pendant vertex, say with $k$ adjacent to $m$, with edge $e_{1}$ between them, and $k$ is adjacent to $l$ with edge $e_{2}$ between them. If $L_{k, k}^{\#}-L_{m, m}^{\#} \geq 0$, then, by (2.3),

$$
\left|\beta_{k}\left(e_{1}\right)\right| \geq\left|\beta_{m}\left(e_{1}\right)\right| \text {. }
$$

But as $\left|\beta_{k}\left(e_{1}\right)\right|+\left|\beta_{m}\left(e_{1}\right)\right|=n$, we find that

$$
\left|\beta_{k}\left(e_{1}\right)\right| \geq \frac{n}{2} .
$$

Now, as $k \in \beta_{k}\left(e_{1}\right) \cup \beta_{k}\left(e_{2}\right)$, we find that

$$
\left|\beta_{k}\left(e_{2}\right)\right| \leq \frac{n-2}{n},
$$

so that $L_{k, k}^{\#}-L_{l, l}^{\#}<0$. Hence $L_{k, k}^{\#}$ cannot be the maximal diagonal entry in $L^{\#}$. It now follows that the maximal diagonal entry must occur at a pendant vertex.

To see that the minimal inverse status number occurs at a centroid, note first that on the one hand vertex $l$ is centroid if and only if

$$
\left|\beta_{l}(e)\right| \leq \frac{n}{2}
$$

for all edges $e$ incident with $l$. On the other hand, because for any adjacent vertices $i$ and $j,\left|\beta_{j}(e)\right|+\left|\beta_{i}(e)\right|=n$, where $e$ is the adjacent edge between $i$ and $j$, in order for vertex $j$ to satisfy that

$$
L_{j, j}^{\#}=\min _{1 \leq i \leq n} L_{i, i}^{\#}
$$


it is necessary, again by (2.3), that $\left|\beta_{j}(e)\right| \leq n / 2$ for all edges incident with $j$. Our proof is now complete.

For each edge $e \in T, T \backslash\{e\}$ has two connected components, $J_{e}$ has $j_{e}$ vertices and $N \backslash J_{e}$ has $n-j_{e}$ vertices.

THEOREM 3.5.

$$
\sum_{i=1}^{n} \tilde{d}_{i}=2 n \sum_{i=1}^{n} L_{i, i}^{\#}=\sum_{e \in T} \frac{2 j_{e}\left(n-j_{e}\right)}{w(e)}
$$

Proof. From the definition of a inverse status number in (1.2) we have that

$$
\sum_{i=1}^{n} \tilde{d}_{i}=\sum_{v \in T} \sum_{u \in T} \sum_{e \in \mathcal{P}_{u, v}} \frac{1}{w(e)}
$$

For each edge $e$, there are $2 j_{e}\left(n-j_{e}\right)$ unordered pairs of vertices $u$ and $v$ such that $e$ is on the path between them. Hence, each edge $e$ contributes $2 j_{e}\left(n-j_{e}\right) / w(e)$ to the above sum so that

$$
\sum_{i=1}^{n} \tilde{d}_{i}=\sum_{e \in T} \frac{2 j_{e}\left(n-j_{e}\right)}{w(e)}
$$

Now from (2.2),

$$
2 n \sum_{i=1}^{n} L_{i, i}^{\#}=\frac{2}{n} \sum_{v \in T} \sum_{e \in T} \frac{\left|\beta_{v}(e)\right|^{2}}{w(e)}=\frac{2}{n} \sum_{e \in T} \sum_{v \in T} \frac{\left|\beta_{v}(e)\right|^{2}}{w(e)} .
$$

If $v \in J_{e}$, then

$$
\left|\beta_{v}(e)\right|^{2}=\left(n-j_{e}\right)^{2}
$$

while if $v \in N \backslash J_{e}$, then

$$
\left|\beta_{v}(e)\right|^{2}=j_{e}^{2}
$$

Consequently,

$$
\begin{aligned}
\sum_{e \in T} \sum_{v \in T} \frac{\left|\beta_{v}(e)\right|^{2}}{w(e)} & =\sum_{e \in T} \frac{1}{w(e)}\left(\sum_{v \in J_{e}}\left|\beta_{v}(e)\right|^{2}+\sum_{v \in N \backslash J_{e}}\left|\beta_{v}(e)\right|^{2}\right) \\
& =\sum_{e \in T} \frac{1}{w(e)}\left(\left(n-j_{e}\right)^{2} j_{e}+j_{e}^{2}\left(n-j_{e}\right)\right) \\
& =\sum_{e \in T} \underset{n j_{e}\left(n-j_{e}\right)}{w(e)} .
\end{aligned}
$$

The result now follows.

We can now give a precise value to the constant $c$ of (3.3).

Corollary 3.6.

$$
\left(L_{1,1}^{\#}, \ldots, L_{n, n}^{\#}\right)=\frac{1}{n}\left(\tilde{d}_{1}, \ldots, \tilde{d}_{n}\right)-\frac{1}{n^{2}} \sum_{e \in T} \frac{j_{e}\left(n-j_{e}\right)}{w(e)} \mathbf{1}=\frac{1}{n}\left(\tilde{d}_{1}, \ldots, \tilde{d}_{n}\right)-\frac{1}{2 n^{2}} \sum_{i=1}^{n} \tilde{d}_{i} \mathbf{1} .
$$


Proof.

$$
c \mathbf{1}=\left(L_{1,1}^{\#}, \ldots, L_{n, n}^{\#}\right)-\frac{1}{n}\left(\tilde{d}_{1}, \ldots, \tilde{d}_{n}\right)
$$

so that

$$
n c=\frac{1}{n} \sum_{e \in T} \frac{j_{e}\left(n-j_{e}\right)}{w(e)}-\frac{2}{n} \sum_{e \in T} \frac{j_{e}\left(n-j_{e}\right)}{w(e)} .
$$

Solving for $c$ now yields the result.

Remark. We comment that as the nonzero eigenvalues of the group inverse of the Laplacian are the reciprocals of the nonzero eigenvalues of the Laplacian, Theorem 3.5 generalizes an equality for the Wiener index of an unweighted tree (see Merris [9, Theorem 5.5] and references cited therein), namely, that

$$
\sum_{i=1}^{n} \tilde{d}_{i}=n \sum_{\lambda \in \sigma(L) \backslash\{0\}} \frac{1}{\lambda},
$$

where $\sigma(\cdot)$ denotes the spectrum of a matrix. $L^{\#}$.

We next develop formulas, in terms of distances, for the off-diagonal entries in

Theorem 3.7. For $i \neq j, 1 \leq i, k \leq n$,

$$
L_{i, n}^{\#}=\frac{\tilde{d}_{i}+\tilde{d}_{k}}{2 n}-\frac{1}{2} \tilde{d}_{i, k}-\frac{1}{2 n^{2}} \sum_{j=1}^{n} \tilde{d}_{j}
$$

Proof. Without loss of generality we can take $k=n$ and $i=1, \ldots, n-1$. First we claim that

$$
L_{i, i}^{\#}-L_{n, n}^{\#}=\tilde{d}_{i, n}-\frac{2}{n} \sum_{e \in \mathcal{P}_{i, n}} \frac{\beta_{n}(e)}{w(e)} .
$$

To see this note that if $e \notin \mathcal{P}_{i, n}$, then its contribution to $L_{i, i}^{\#}$ is the same as that to $L_{n, n}^{\#}$. On the other hand, if $e \in \mathcal{P}_{i, n}$, the contribution of $e$ to $L_{n, n}^{\#}$ is

$$
\frac{1 \beta_{n}(e)}{n w(e)},
$$

while its contribution to $L_{i, i}^{\#}$ is

$$
\frac{1 n-\beta_{n}(e)}{n} w(e) .
$$

Thus

$$
L_{i, i}^{\#}-L_{n, n}^{\#}=\frac{2}{n} \sum_{e \in \mathcal{P}_{i, n}} \frac{n-\beta_{n}(e)}{w(e)}=\tilde{d}_{i, n}-\frac{2}{n} \sum_{e \in \mathcal{P}_{i, n}} \frac{\beta_{n}(e)}{w(e)},
$$

as desired. Hence, for $1 \leq j \leq n$, we have that

$$
L_{j, j}^{\#}=\tilde{d}_{j, n}-\frac{2}{n} \sum_{f \in \mathcal{P}_{i, n}} \frac{\beta_{n}(f)}{w(f)},
$$


and this yields that

$$
\begin{aligned}
L_{i, i}^{\#}-L_{j, j}^{\#} & =\tilde{d}(i, n)-\tilde{d}(j, n)+2\left[\frac{1}{n} \sum_{f \in \mathcal{P}_{j, n}} \frac{\beta_{n}(f)}{w(f)}-\frac{1}{n} \sum_{f \in \mathcal{P}_{i, n}} \frac{\beta_{n}(e)}{w(e)}\right] \\
& =\tilde{d}(i, n)-\tilde{d}(j, n)+2\left(L_{i, n}^{\#}-L_{n, n}^{\#}\right) .
\end{aligned}
$$

It now follows that for some constant $\alpha$,

$$
2 L_{i, n}^{\#}=L_{i, i}^{\#}-\tilde{d}(i, n)+\alpha, \quad 1 \leq i \leq n-1 .
$$

To find $\alpha$, note that

$$
\begin{aligned}
(n-1) \alpha & =2 \sum_{i=1}^{n-1} L_{i, n}^{\#}-2 \sum_{j=1}^{n-1} L_{j, n}^{\#}+\sum_{i=1}^{n-1} \tilde{d}(i, n) \\
& =-2 L_{n, n}^{\#}-\sum_{i=1}^{n-1} L_{i, i}^{\#}+\tilde{d}_{n}=-L_{n, n}^{\#}-\sum_{i=1}^{n} L_{i, i}^{\#}+\tilde{d}_{n} \\
& =-\frac{1}{n} \tilde{d}_{n}+\frac{1}{2 n^{2}} \sum_{j=1}^{n} \tilde{d}_{j}-\frac{1}{2 n} \sum_{j=1}^{n} \tilde{d}_{j}+\tilde{d}_{n} \\
& =\frac{n-1}{n} \tilde{d}_{n}-\frac{n-1}{2 n^{2}} \sum_{j=1}^{n} \tilde{d}_{j} .
\end{aligned}
$$

Thus we have that

$$
\alpha=\frac{1}{n} \tilde{d}_{n}-\frac{1}{2 n^{2}} \sum_{j=1}^{n} \tilde{d}_{j},
$$

which yields

$$
\begin{aligned}
L_{i, n}^{\#} & =\frac{1}{2}\left(L_{i, i}^{\#}-\tilde{d}(i, n)+\frac{1}{n} \tilde{d}_{n}-\frac{1}{2 n^{2}} \sum_{j=1}^{n} \tilde{d}_{j}\right) \\
& =\frac{1}{2}\left[\frac{1}{n}\left(\tilde{d}_{i}+\tilde{d}_{n}\right)-\tilde{d}(i, n)-\frac{1}{n^{2}} \sum_{j=1}^{n} \tilde{d}_{j}\right],
\end{aligned}
$$

as desired.

In Deutsch and Neumann [5], the following problem was posed: characterize the set of all $n \times n$ irreducible singular $M$-matrices whose group inverse is also an $M$ matrix. In the circumstances of this paper we have the following result.

Corollary 3.8. Let $L$ be the Laplacian of a weighted tree on $n$ vertices. Then $L^{\#}$ is an $M$-matrix if and only if for every pair of adjacent vertices $i$ and $j$ we have that

$$
\tilde{d}_{i}+\tilde{d}_{j} \leq \frac{n}{w\left(e_{i, j}\right)}+\frac{1}{n} \sum_{k=1}^{n} \tilde{d}_{k} .
$$


Proof. For vertices $a$ and $b$ with $1 \leq a, b \leq n-1$, we have from (2.4) that

$$
L_{a, n}^{\#}-L_{b, n}^{\#}=\frac{1}{n}\left(\sum_{f \in \mathcal{P}_{b, n}} \frac{\beta_{n}(f)}{w(f)}-\sum_{e \in \mathcal{P}_{a, n}} \frac{\beta_{n}(e)}{w(e)}\right),
$$

so we see that, in the $n$th row of $L^{\#}$, the entries are decreasing along paths away from $n$. Hence $L^{\#}$ is an $M$-matrix if and only if for any adjacent vertices $i$ and $j$ we have that $L_{i, j}^{\#} \leq 0$. Imposing this condition in (3.4) now yields the result.

For unweighted trees we can determine precisely which trees admit a Laplacian whose group inverse is an $M$-matrix. For this purpose we require the following lemma.

Proposition 3.9. Let $T$ be an unweighted tree on $n$ vertices. Then the maximum of $\tilde{d}_{i}+\tilde{d}_{j}$ over all pairs of adjacent vertices $i$ and $j$ occurs for the adjacent vertices of some pendant edge.

Proof. Let $i$ and $j$ be adjacent vertices with $\tilde{d}_{i}+\tilde{d}_{j}$ maximal. Assume that neither $i$ nor $j$ is a pendant vertex. Then there exist vertices $h \neq j$ and $k \neq i$ such that $h$ is adjacent to $i$ and $k$ is adjacent to $j$. Let $\alpha, \beta, \gamma$, and $\delta$, respectively, be the set of vertices $v$ for which the path from $v$ to the path $h-i-j-k$ ends at $h, i, j$, and $k$, respectively.

Note that the distance from a vertex in $\gamma$ to either $i$ or $k$ is equal, the distance from a vertex in $\delta$ to $i$ is 2 more than its distance to $k$, and the distance from a vertex in $\alpha \cup \beta$ to $k$ is 2 more than its distance to $i$. Thus,

$$
\tilde{d}_{i}-\tilde{d}_{k}=2(|\delta|-|\alpha|-|\beta|) .
$$

Similarly,

$$
\tilde{d}_{j}-\tilde{d}_{h}=2(|\alpha|-|\gamma|-|\delta|) .
$$

Since $\tilde{d}_{i}+\tilde{d}_{j}$ is maximal, it follows that $\tilde{d}_{i}-\tilde{d}_{k} \geq 0$ and $\tilde{d}_{j}-\tilde{d}_{h} \geq 0$. Adding equations (3.5) and (3.6) yields

$$
0 \leq-2(|\beta|+|\gamma|)
$$

This implies that $\beta$ and $\gamma$ are empty. However, this contradicts the fact that $i \in \beta$ and $j \in \gamma$. We conclude that either $i$ or $j$ is a pendant vertex.

We can now show that for unweighted trees, the only trees whose Laplacian has a group inverse which is an $M$-matrix are the stars of all orders.

TheOREM 3.10. Let $L$ be the Laplacian of a tree $T$ with $n$ vertices. Then $L^{\#}$ is an $M$-matrix if and only if $T$ is a star.

Proof. By Corollary 3.8,

$$
d_{i}+d_{j} \leq n+\frac{1}{n} \sum_{k=1}^{n} \tilde{d}_{k}
$$

for each pair of adjacent vertices $i$ and $j$. Let $i$ be a pendant vertex and $j$ the vertex adjacent to $i$, and let $e$ be the edge joining $i$ and $j$. By counting the contributions of each edge of $T$ to $\tilde{d}_{i}+\tilde{d}_{j}$ and to $\sum_{k=1}^{n} \tilde{d}_{k}$ we see that

$$
\tilde{d}_{i}+\tilde{d}_{j}=n+2 \sum_{f \neq e}\left|\beta_{i}(f)\right|
$$


and

$$
\sum_{k=1}^{n} \tilde{d}_{k}=2(n-1)+2 \sum_{f \neq e}\left|\beta_{i}(f)\right|\left(n-\left|\beta_{i}(f)\right|\right) .
$$

Substituting into (3.7) and simplifying we obtain that

$$
\sum_{f \neq e} \beta_{i}(f)^{2} \leq n-1
$$

Since $\beta_{i}(f) \geq 1$ for $f \neq e$, we conclude that $\beta_{i}(f)=1$ for $f \neq e$. It now follows that $T$ is a star with center $j$.

We note that in Chen, Kirkland, and Neumann [4, Corollary 5.4] it is shown that if $n \geq 5$, then no weighted path of order $n$ can yield a Laplacian whose group inverse is an $M$-matrix. The following example, taken from [4], exhibits a weighted path of order 4 whose group inverse is an $M$-matrix. Let

$$
L=\left[\begin{array}{cccc}
0.2 & -0.2 & 0 & 0 \\
-0.2 & 0.6 & -0.4 & 0 \\
0 & -0.4 & 0.6 & -0.2 \\
0 & 0 & -0.2 & 0.2
\end{array}\right]
$$

Then

$$
L^{\#}=\left[\begin{array}{cccc}
3.75 & 0.000 & -1.25 & -2.5 \\
0.000 & 1.250 & 0.000 & -1.250 \\
-1.25 & 0.000 & 1.25 & 0.000 \\
-2.50 & -1.25 & 0.000 & 3.75
\end{array}\right]
$$

\section{REFERENCES}

[1] A. Berman and R. J. Plemmons, Nonnegative Matrices in the Mathematical Sciences, SIAM, Philadelphia, PA, 1994

[2] S. L. Campbell and C. D. Meyer, JR., Generalized Inverses of Linear Transformations, Dover Publications, New York, 1991.

[3] S. Chaiken, A combinatorial proof of the all minors matrix tree theorem, SIAM J. Alg. Disc. Meth., 3 (1982), pp. 319-329.

[4] Y. Chen, S. J. Kirkland, and M. Neumann, Group generalized inverses of M-matrices associated with periodic and nonperiodic Jacobi matrices, Linear and Multilinear Algebra, 39 (1995), pp. 325-340.

[5] E. Deutsch and M. Neumann, Derivatives of the Perron root at an essentially nonnegative matrix and the group inverse of an M-matrix, J. Math. Anal. Appl., 102 (1984), pp. 1-29.

[6] F. HARARY, Status and contrastatus, Sociometry, 22 (1959), pp. 23-43.

[7] S. J. Kirkland, M. Neumann, And B. Shader,.Bounds on the subdominant eigenvalue involving group inverses with applications to graphs, Czechoslovakia Math. J., to appear.

[8] S. J. Kirkland, M. Neumann, and B. Shader, Characteristic vertices of weighted trees via perron values, Linrear and Multilinear Algebra, 40 (1996), pp. 311-325.

[9] R. MerRis, Laplacian matrices and graphs: A survey, Linear Algebra Appl., 197, 198 (1994), pp. 143-176.

[10] S. PARTer AND J. W. T. Youngs, The symmetrization of matrices by diagonal matrices, J. Math. Anal. Appl., 4 (1962), pp. 102-110. 